\title{
MÉTODO CANGURU: PRÁTICAS INVESTIGATIVAS E DE CUIDADO DE ENFERMAGEM NO MODELO DE ADAPTAÇÃO DE ROY
}

\author{
Kangaroo method: investigative practices and nursing care in the roy adaptation model \\ Método canguro: prácticas de investigación y de atención en enfermería en el modelo \\ de adaptación de roy
}

Márcia Borck ${ }^{1}$

Evanguelia Kotzias Atherino dos Santos ${ }^{2}$

\section{RESUMO}

Trata-se de uma pesquisa convergente-assistencial, de natureza qualitativa, que teve como objetivos: investigar a experiência no processo de adaptação de seis famílias na terceira etapa do Método Canguru com recém-nascidos pré-termo e/ou de baixo peso, no período de outubro/06 a fevereiro/07; e implementar a consulta de enfermagem dentro dos quatro modos adaptativos da Teoria de Roy. A coleta de dados foi realizada através do processo de cuidar do modelo adaptativo de Roy, utilizando as técnicas de observação e da entrevista semiestruturada. 0 processo de análise ocorreu por meio da apreensão, síntese, teorização e transferência. Os resultados mostram a necessidade de fortalecer o papel da família na desospitalização e a comunicação entre a equipe interdisciplinar e rever critérios de alta da terceira etapa. Este estudo evidencia a terceira etapa como oportunidade para retroalimentar o sistema de cuidado e apoiar as famílias para sobreporem seus conflitos, preocupações, medos, inseguranças e o próprio comportamento imaturo do bebê.

Palavras-chave: Enfermagem neonatal. Recém-nascido prematuro. Recém-nascido de baixo peso. Relações mãe-filho.

\begin{abstract}
This study deals with a qualitative, Convergent-Care Research which objectives were to investigate the adaptation process experienced by six families in this third stage of the Kangaroo Method (KM) with premature and/or low weight newborns, from 0ct/06 to Feb/07, and implement a nursing visit with each participant of the study, in reference to the four adaptive modes of the Roy Theory. The Adaptation Model proposed by Sister Callista Roy was used as a theoretical reference. The data was collected through Roy's adaptive care process model, applying observation techniques and semi-structured interviews. The data analysis process occurred through apprehension, synthesis, theorization, and transference. The results show the need to strengthen the role of the family when release from the hospital, communication among the interdisciplinary team, reviewing the criteria for release in the third stage, as well as guaranteeing all the possibilities for continued care. This study evidences the third stage as an opportunity to reinforce the care system, support for the family in order to overcome their conflicts, concerns, fears, insecurities, and the very immature behavior of the baby.
\end{abstract}

Keywords: Neonatal nursing. Infant, premature. Infant, low birth weight. Mother-child relations.

\section{Resumen}

Se trata de una Investigación Convergente y Asistencial, cualitativa. Sus objetivos fueron investigar la experiencia en el proceso de adaptación de seis familias en la tercera etapa del Método Canguro con recién nacidos pre término y/o de bajo peso, en el periodo de Octubre $/ 06$ a Febrero /07 e implementar la consulta de enfermería dentro de los cuatro modos adaptativos de la Teoría de Roy. La recolección de los datos fue realizada a través del proceso de cuidar del modelo adaptativo de Roy, utilizando las técnicas de observación y la entrevista parcialmente estructurada. El proceso de análisis se hizo por medio de la aprehensión, síntesis, teorización y transferencia. Los resultados muestran la necesidad de fortalecer el papel de la familia en la deshospitalización, la comunicación entre el equipo disciplinario y rever criterios de alta de la tercera etapa. En este estudio se evidencia la tercera etapa como una oportunidad para retroalimentar el sistema de cuidado, apoyar las familias para sobreponer sus conflictos, preocupaciones, miedos, inseguridades y el propio comportamiento inmaduro del bebé.

Palabras-Clave: Enfermería neonatal. Prematuro. Recién nacido de bajo peso. Relaciones madre-hijo.

\footnotetext{
Mestre em Enfermagem, Enfermeira do Serviço de Neonatologia do Hospital Universitário da UFSC, Consultora para o Método Canguru no Brasil do Ministério da Saúde, Florianópolis - SC. Brasil. E-mail: marcia.huufsc@yahoo.com.br; ${ }^{2}$ Doutora em Enfermagem, Professora Associada do Departamento de Enfermagem e do Programa de Pós-Graduação em Enfermagem da UFSC, Líder do Grupo de Pesquisa em Enfermagem na Saúde da Mulher e do RecémNascido, Florianópolis - SC. Brasil. E-mail: gregos@matrix.com.br
} 


\section{INTRODUÇÃO}

Ao entrar pela primeira vez no Serviço de Neonatologia do Hospital Universitário (HU) da Universidade Federal de Santa Catarina (UFSC), sentimo-nos tão frágeis quanto os pequeninos seres que vimos dentro das incubadoras ou berços aquecidos. Recém-nascidos pré-termo e/ou de baixo peso, tratando de ganhar peso e conquistando sua autonomia, sob atenção dos profissionais de saúde e o olhar terno e atentivo dos pais. Começava ali uma grande descoberta, pois, em um desafio constante, tivemos que desenvolver competências e habilidades para atuar nesta especialidade. Precisamos despertar vigília diferenciada, deixar aflorar nossa sensibilidade para um ser humano que nos apresentava toda sua vulnerabilidade e dependência, o qual também precisava dos nossos cuidados para sua adaptação ao meio ambiente e para apoio aos seus pais.

Nossa escolha para o desenvolvimento deste estudo emergiu da prática profissional buscando uma nova leitura de nossas vivências e interações com o recém-nascido pré-termo e/ou de baixo peso, o que nos motivou a teorizar pela aplicação do Modelo de Adaptação de Roy na terceira etapa do Método Canguru (MC). Tal motivação se deu pela tênue passagem da atuação como cuidadores principais do cuidado, até então promovido por nós profissionais, para os pais e familiares, buscando também contribuir com a produção científica nesta área.

A primeira e a segunda etapas do Método Canguru redesenharam o cuidado na fase intra-hospitalar. A terceira etapa se inicia com a alta hospitalar, e exige acompanhamento ambulatorial criterioso do bebê e de sua família até atingir o peso de $2.500 \mathrm{~g}$, dando continuidade à abordagem biopsicossocial. As atribuições nesta etapa são: exame físico completo da criança, tomando como referências básicas o grau de desenvolvimento, o ganho de peso, 0 comprimento e o perímetro cefálico, e levando em conta a idade gestacional corrigida; avaliar o equilíbrio psicoafetivo entre a criança e a família e oferecer o devido suporte; apoiar a manutenção de rede social de apoio; corrigir as situações de risco, como ganho inadequado de peso, sinais de refluxo, infecção e apneias; orientar e acompanhar tratamentos especializados; orientar esquema adequado de imunizações. ${ }^{1}$

É dado o privilégio, a todos nós, que trabalhamos no ambiente neonatal, de acompanhar os primeiros encontros entre um recém-nascido (RN) e sua família. São nossos olhos que percebem as primeiras tentativas de proximidade, somos os primeiros a escutar os comentários em voz baixa dos pais sobre o bebê na incubadora, são nossos atos que podem facilitar a dinâmica e o movimento desses encontros. Assim, assumir esses novos paradigmas é para nós, cuidadores profissionais, a possibilidade de oferecer aos bebês e aos pais um espaço facilitador para a formação de seus vínculos.?
Há ainda uma lacuna no que diz respeito à sistematização da assistência de enfermagem durante a promoção e a prática do método, uma vez que o método em si não contempla o processo de cuidar em enfermagem. ${ }^{3}$

Embora o MC tenha sido recomendado, em 2000, para ser implementado como um método assistencial nas instituições do Sistema Único de Saúde, poucos estudos brasileiros foram publicados sobre o modo como esse método vem sendo adotado pelas maternidades brasileiras, em suas três etapas. ${ }^{4}$

Estudos mostram a importância de programas interdisciplinares preventivos de follow up de prematuros, especialmente no $1^{\circ}$ ano de vida, com intuito de promover 0 desenvolvimento da criança e minimizar as possíveis sequelas de saúde do período neonatal. 5,6,7,7,9 Serviços de saúde e das redes familiares no sucesso do MC são pesquisados na fase hospitalar e domiciliar. Na maioria dos casos, a adaptação à rotina familiar não teve implicações negativas para a saúde do bebê. Em casa entram em jogo outros fatores, como a presença do companheiro e de outros filhos e as tarefas domésticas. ${ }^{10}$

A família é considerada uma das entidades sociais mais relevantes e significativas para a sociedade, e atualmente está entre as mais estudadas, em decorrência, por exemplo, de sua importância para o cultivo de valores e crenças que contribuem para a formação dos membros que a constituem. As experiências vivenciadas nessa organização social contemplam um viver e conviver de singularidades que, quando somadas, retratam sua força, limites e enfrentamentos. ${ }^{11}$ Os pais de prematuros são considerados um grupo de risco, pois já ficam debilitados com o sofrimento advindo das complicações da gravidez. Assim, encontram-se prejudicados na autoconfiança e, consequentemente, na capacidade de criar esse filho. ${ }^{12}$

Conhecer como as famílias lançam seu olhar para o contexto de vida com o nascimento prematuro de seu filho e o desenvolvimento de suas competências para o cuidado nos permitem avaliar nossas intervenções. Deste modo, a intenção com este estudo foi aliar o planejamento, a aplicação e a análise da sistematização do cuidado de enfermagem com um percurso investigativo, o que possibilitou o surgimento de algumas respostas às inquietações deste mesmo processo de cuidar.

0 Modelo da Adaptação proposto por Roy inclui a noção de estímulos e respostas. Os estímulos pontuam a variação de respostas que são exclusivas a cada pessoa. Dentre os estímulos significativos encontram-se o grau de mudança, as experiências anteriores, o nível de conhecimento, os pontos fortes e/ou as

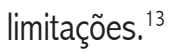

Por reconhecermos a terceira etapa como a que dá continuidade à assistência ao recém-nascido pré-termo e/ou de baixo peso após a alta, exigindo diferentes modos adaptativos de todos os envolvidos, optamos por realizar o presente estudo que tem como objetivos:

- Investigar a experiência no processo de adaptação de famílias na terceira etapa do MC com recém-nascidos pré-termo e/ou de baixo peso. 
- Implementar a consulta de enfermagem com cada participante do estudo referente aos quatro modos adaptativos da Teoria de Roy.

\section{METODOLOGIA}

Trata-se de uma pesquisa convergente-assistencial (PCA), qualitativa, tendo o Modelo de Adaptação de Roy como referencial teórico. A PCA articulou teoria e prática na construção do conhecimento. Esta modalidade de pesquisa procura manter, em todas as fases do processo investigativo, uma estreita relação com a prática assistencial, tendo como finalidade "encontrar alternativas para solucionar ou minimizar problemas, realizar mudanças e introduzir inovações na prática". ${ }^{14}$

Este estudo foi desenvolvido na unidade de neonatologia da maternidade do Hospital Universitário Polydoro Ernani de São Thiago da Universidade Federal de Santa Catarina HU/UFSC. Os sujeitos significativos foram seis mães e familiares com seus bebês recém-nascidos pré-termo e/ou de baixo peso internados na referida unidade e que ingressaram na terceira etapa do MC durante o período de out/ 06 a fev/07, incluindo somente os residentes na grande Florianópolis. Foi rigorosamente considerado o consentimento livre e esclarecido das mães e familiares em participar do estudo.

0 tamanho da amostra não foi determinada antes de iniciar a coleta de informações, pois dependia dos depoimentos apreendidos pelos entrevistados (mães e familiares). A amostra foi definida à medida que as informações foram sendo saturadas, ficando desta forma condicionada à compreensão do fenômeno, ou seja, quando iniciasse a repetição dos discursos, não surgindo novas informações.

Conceituamos os familiares como seres biopsicossociais, singulares, unidos por laços de consanguinidade ou afetividade, que vivenciam e compartilham a mesma experiência envolvendo recém-nascidos pré-termo e de baixo peso em terceira etapa do Método Canguru, formadores da sua rede de suporte social.

A coleta de dados foi realizada através do processo de cuidar do modelo adaptativo de Roy inserido na terceira etapa do $M C$, sendo este ajustado a partir do processo de enfermagem proposto por Roy. ${ }^{13} \mathrm{~A}$ primeira fase foi a de coleta de dados por observação e entrevista. A técnica principal utilizada foi a observação participante, pela sua propriedade de captar uma variedade de fenômenos de uma situação social, os quais não podem ser obtidos unicamente por meio de entrevistas. ${ }^{15}$

Na sequência foi realizada a entrevista individual na qual ocorre interação face a face com o entrevistado, na modalidade de entrevista semiestruturada que parte de tópicos relacionados ao tema de pesquisa.

Utilizamos gravaç̃̃es como instrumentos de registro e transcrição das fitas com a devida autorização dos participantes. 0 anonimato dos sujeitos da pesquisa foi assegurada pela numeração que identifica bebê, mãe e pai. Esta surgiu da soma numerológica da data de nascimento do bebê com seu nome completo, e a sequência da apresentação seguiu a ordem de ingresso na terceira etapa do Método Canguru.

Foi também utilizado um diário de campo, elaborado após cada encontro, no qual registramos dados relativos a cada vivência, incluindo a presença e envolvimento dos participantes no processo através da comunicação verbal e não verbal, bem como nossas reflexões pessoais. Observamos também informações registradas em prontuário para identificar dados maternos e paternos, dados do parto e do recém-nascido prétermo e/ou de baixo peso durante sua internação na unidade de neonatologia até seu ingresso na terceira etapa do Método Canguru.

No processo analítico, utilizamos os passos propostos por Morse e Field ${ }^{14}$, composto pelos seguintes processos: apreensão, síntese, teorização e transferência. Esta pesquisa respeitou os princípios de beneficência, não maleficência, justiça e autonomia, preconizados pela Resolução no 196/96 do Conselho Nacional de Saúde e os princípios do Código de Ética profissional. Foi submetida ao Comitê de Ética em Pesquisa da UFSC, sendo aprovada sob o parecer n 237/2006.

\section{REFERÊNCIAL TEÓRICO}

Percebemos que a utilidade do Modelo de Adaptação de Roy para a pesquisa, a educação, a administração e a prática de enfermagem está bem documentada, fato que reforçou nosso interesse em adotá-lo.

Para operacionalizar o referido modelo na prática assistencial, Roy propõe a utilização do processo de enfermagem contemplando seis etapas: avaliação de comportamento, avaliação de estímulos, diagnóstico de Enfermagem, estabelecimento de metas, intervenção e avaliação. ${ }^{13}$

0 modelo proposto por Roy inclui a noção de estímulos e respostas. Entre os estímulos significativos, encontram-se 0 grau de mudança, as experiências anteriores, o nível de conhecimento, os pontos fortes e/ou as limitações. ${ }^{13}$

Ao buscarmos identificar as percepções de mães e familiares que acompanharam seus bebês em terceira etapa do Método Canguru, imergiram diferentes culturas que em uma ação de reciprocidade influenciaram no cuidado adaptativo ao recém-nascido pré-termo e/ou de baixo peso. E, nessa troca de experiências humanas, promovemos a inserção da enfermeira, estabelecendo o seu papel na terceira etapa do Método Canguru.

Após a fase de entrevista criamos as categorias diagnósticas, buscando investigar os indicadores de adaptação positiva e os problemas comuns de adaptação nos quatro modos de adaptação descritos por Roy (Fisiológico, Autoconceito, Função do papel e Interdependência). Para tanto, observamos os comportamentos dos envolvidos e as respostas das mães e familiares junto às do seu bebê diante do cuidado realizado por eles durante a terceira etapa. Registramos como estavam realizando sua adaptação em ambiente domiciliar por meio de seus depoimentos e questionamentos, bem como seu 
envolvimento a partir das falas e olhares durante a consulta médica e, posteriormente, a consulta de enfermagem.

$\mathrm{Na}$ sequência ao processo de cuidar do modelo adaptativo de Roy, construímos o diagnóstico de enfermagem. Identificando as adaptações positivas, estas foram estimuladas se necessárias ao processo de cuidar, e os problemas de adaptação que exigiam articulações do enfermeiro receberam atenção espećífica.

Com o diagnóstico de enfermagem elaborado, passamos para a descrição dos cuidados de enfermagem, estabelecendo as metas com clareza nos resultados que esperávamos alcançar. Este plano foi estabelecido com a mãe, pai e familiares para os comportamentos a serem alcançados por eles e seu bebê.

Com as metas estabelecidas, e buscando reconhecer como alcançá-las, realizamos a avaliação.

No controle sistemático sugerido pelo Ministério da Saúde, ${ }^{1}$ observamos, para fins de avaliação do processo da prática, o trabalho com a demanda da família em relação à avaliação pôndero-estatural, exame clínico sumário, avaliação do equilibrio psicoafetivo mãe-bebê, diagnóstico, tratamento e orientação, promoção do aleitamento materno, imunização, revisão do sumário de alta e atenção para controles especiais. 0 processo avaliativo transcorreu sempre no final de cada retorno em terceira etapa do Método Canguru.

\section{RESULTADOS E DISCUSSÃO}

No processo de cuidar, abstraímos lições de vida das mães e familiares, de enfrentamento, de adaptações originadas por estímulos, ora focais, ora contextuais, em um processo de retroalimentação complexo como vivenciamos ser a teoria de adaptação de Roy. A teórica lança desafios de criatividade para nós enfermeiros e de apuração do olhar para o cuidar do ser humano além do físico e do verbalizado, mas conduz sabiamente a nossa interação no processo como ar ticuladores para o bem-estar, promovendo a integridade da pessoa no momento que busca, ou não, a adaptação.

A cada aproximação nossa com as famílias, revalorizamos 0 ato de escutar e nos realizamos com a naturalidade com a qual buscam aprender através da expressão de sentimentos ambíguos, como medo ou felicidade, e então pontuamos o cuidado na demanda necessária. Os sujeitos envolvidos nos escutam e absorvem conhecimento até do nosso olhar, assim desenvolvem potencialidades inatas ou adormecidas. Pai, mãe e familiares não procuram o isolamento. Eles, na terceira etapa, em que são os atores principais, querem desenvolver competências e focam a enfermeira nesta busca, e isto é gratificante, muitas vezes trabalhoso, mas sempre gratificante. É sentir de fato a vibração do cuidado adaptativo.

No momento em que nos permitimos aceitações, por exemplo, de valores trazidos pelas mães e familiares, exercitamos o apoio, mas percebemos que, neste exato momento, inseríamos a articulação de papéis, construção de metas e avaliação conjunta proposta por Roy e o resultado foi um só: interação e crescimento.

Apresentamos na Figura 1, a seguir, uma representação diagramática baseada nos registros dos sistemas adaptativos humanos. 0 diagrama criado relaciona os subsistemas envolvidos na terceira etapa do MC.

Figura 1 -Representação sobre relacionamento dos elementos adaptativos no sistema humano envolvido na terceira etapa do MC segundo os elementos conceituais da Teoria de Adaptação. ${ }^{13}$

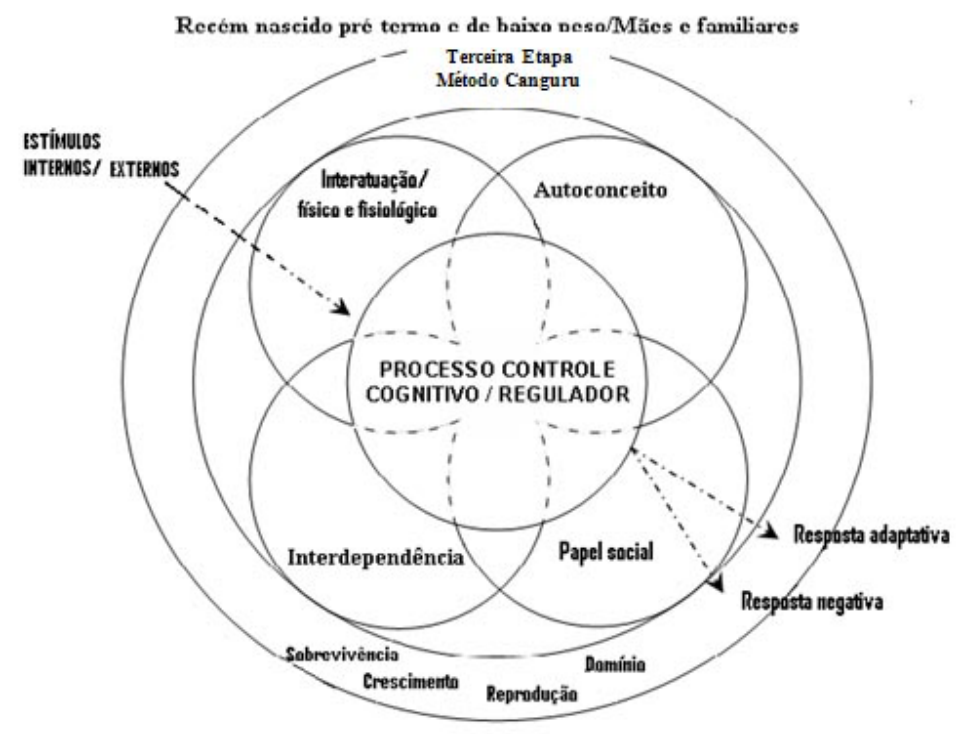


Conforme os recém-nascidos acompanhados neste estudo, na realidade do HU/UFSC, o peso dos bebês na alta é de $1.750 \mathrm{~g}$ a $1.900 \mathrm{~g}$, a idade gestacional corrigida é de $34 \mathrm{~s} 1 \mathrm{~d} \mathrm{a}$ $39 \mathrm{~s} 1 \mathrm{~d}$, mamando ao peito e em alguns casos com complemento. 0 primeiro retorno acontece dentro de 72 a 120 horas, e a frequência das consultas é de duas por semana até o peso de $2.000 \mathrm{~g}$. Estes dados são registrados em formulário específico da terceira etapa, mas sentimos falta de mais riquezas nas anotações que nos permitam uma leitura mais próxima da realidade de cada família.

Propomos, assim, recriar o formulário inserindo dados como realização do Canguru ( $<6$ horas/dia -6 a 12 horas/dia -> 12 horas/dia); ganho ponderal diário; condutas em curso (medicações); imunizações; rede social de apoio; visita domiciliar e, na alta, identificar o ambulatório de desenvolvimento (alto risco ou baixo risco).

A família tem expectativas de encontrar um profissional que the ajude a compreender os momentos difíceis, que demandam ações, sentimentos e pensamentos, nem sempre positivos. Com a orientação, a ajuda e o acolhimento da enfermagem, os familiares poderão ultrapassar suas expectativas, possibilitando seu crescimento individual e maior aquisição de habilidades em qualidades humanas. ${ }^{16}$

Buscando superar o mero tecnicismo, Roy desenvolveu uma associação entre um modelo de cuidado como fenômeno da enfermagem e sua inerente condição técnica, em torno de suposições filosóficas baseadas na relação enfermeiro-paciente refletidas na perspectiva da liberdade do indivíduo e no realce à adaptação da pessoa e da saúde na integralidade e totalidade. ${ }^{17}$

Assim, das vivências das famílias observadas neste estudo, em relação ao cuidado adaptativo de seus recémnascidos pré-termo e/ou de baixo peso, apresentamos três categorias primárias em que fazemos uma discussão das representações que convergiram do meio-tempo entre a desospitalização precoce e o primeiro retorno na terceira etapa do MC, e analisamos as vivências que contribuíram com a adaptação do cuidado e as que provocaram circunstâncias (des)adaptativas nas vivências dos sujeitos deste estudo.

Foram identificadas: cinco categorias convergentes do meio-tempo entre a desospitalização precoce e o primeiro retorno na terceira etapa: Valorando o aleitamento materno; Percebendo necessidades de manejo com RN; A felicidade e o medo ao sair do hospital; Primeira noite nenhuma mãe dorme; Reconhecendo e ativando cuidados.

Cinco categorias adaptativas convergentes dos retornos na terceira etapa: Mamãe eu quero mamar; Meu bebê quer isso, quer aquilo. Precisa disso; Meu bebê está crescendo; Nosso bebê, nossa família: é bom estarmos juntos; e Coração tranquilo.

Cinco categorias (des)adaptativas convergentes dos retornos na terceira etapa: Meu leite é pouco, meu peito dói; Para que esses exames/por que esses acompanhamentos; Eu preciso de um especialista; 0 que me fez descuidar do bebê: minha cabeça ou meu coração; e Amar e estar junto, ou só estar junto.

Os dados mais relevantes destas categorizações e que foram trabalhados durante este estudo são descritos a seguir.

A posição canguru não foi percebida por todas as seis famílias deste estudo como um cuidado promotor de desenvolvimento do bebê ou do afeto entre 0 binômio que 0 realiza, mas foi resgatada enquanto meta construída em conjunto nas consultas de enfermagem, tendo sido realizada por todas as mães, 4 pais, 1 avó, 2 irmãs e 1 tio.

A cuidadora principal, a mãe, também se percebe de forma diferenciada na terceira etapa, busca orientações para sua alimentação, cuidados com as mamas, métodos contraceptivos ou tratamentos ginecológicos específicos. Percebemos que as mães sabem que o seu filho será cuidado, enquanto internado, independente do seu estado físico ou emocional, mas na terceira etapa precisam investir para o seu bem-estar, integralmente, pois o cuidado adaptativo acontece essencialmente por meio dela.

0 apoio às mães em necessidades de exames complementares de seus filhos exigiu trabalhoso estímulo contextual, o que foi percebido por elas através do processo educativo sobre triagem neonatal e por se sentirem amparadas durante suas oscilações emocionais, alterando estímulos que contribuíram para comportamentos ineficazes. Acreditamos que tenha ocorrido uma adequação afetiva que, pelas interrelações, insere a pessoa como um ser social.

0 medo, a preocupação trazida pelas mães diante dos controles especiais nos remeteu a uma "leitura de entrelinhas". As mães sempre procuram uma falha em si como causa para as alterações no seu filho. Como exemplo, temos a mãe que associou a alteração hormonal em seu bebê com a opção injetável de seu anticoncepcional, o que lhe mobilizou o sentimento de culpa.

No entanto, mesmo com ambiguidade de sentimentos, eles retornam abertos às consultas em terceira etapa, oportunizam aproximação e compartilham sua arte no cuidado adaptativo, contam estórias e histórias, nos enriqueceram nas suas respostas às inquietações iniciais quando nos envolvemos com este estudo.

Como é possível observar nos resultados desta pesquisa, há casos especiais em que a mãe, o pai e o bebê ainda necessitam de acompanhamento no processo de amamentação, no cuidado canguru, de suporte psicoafetivo e na organização de papéis.

Os resultados mostram a necessidade de fortalecer 0 papel da família na desospitalização, a comunicação entre a equipe interdisciplinar, rever critérios de alta da terceira etapa e garantir todas as possibilidades de atendimento continuado.

A adaptação no ambiente domiciliar varia de acordo com os cuidados e papéis diários assumidos pela mãe e 
familiares e pelos resultados obtidos nas consultas em terceira etapa. Sentimentos ambíguos como medo, ansiedade, preocupação, incertezas afetam o autoconceito e 0 funcionamento familiar, pois mobilizam-se, muitas vezes inconscientemente, por sentirem-se ligados a uma criança que poderá morrer a qualquer momento.

\section{CONSIDERAÇÕES FINAIS}

0 Modelo de Adaptação de Roy renovou nossa dimensão educacional permitindo-nos criar uma metodologia própria, prática e incomparável diante do exercício da percepção do ambiente, ser humano, enfermagem, saúde-doença, adaptação e MC. 0 marco conceitual elaborado inseriu um exercício profissional desafiador, instigante e apaixonante. Ao desvendar leituras e sustentar o referencial teórico sistematizado, ocorreu a conexão entre nossas expectativas enquanto enfermeiras assistenciais e nossas crenças pessoais. A busca pela sobrevivência percebida durante o estudo, que sustenta os esforços da mãe e familiares de um recém-nascido de baixo peso para permanecer como um sistema adaptativo vivo, que passa pela experiência de ter um filho pré-termo e/ou de baixo peso, pode ser classificada como uma resposta adaptativa ou resposta ineficiente. A pesquisa convergente-assistencial foi uma feliz escolha para o percurso metodológico, contribuiu para recriar ações e renovar pensamentos na prática e na pesquisa, pois de fato nos fez vivenciar movimentos de aproximação com os sujeitos, o ambiente e, primordialmente, com o cuidado.

A adoção do Método Canguru visa, em essência, a mudança de atitudes em relação ao cuidado e manuseio do bebê e à participação de sua família. Esse objetivo, por sua vez, tem embutido em si mesmo algumas questões do saber/ fazer do profissional, como a abordagem comunicativa e criativa, a abertura de espaços e a própria relação estabelecida para o cuidado. ${ }^{18}$

0 papel da enfermeira na terceira etapa do $M C$ vai além do supervisionar, coordenar, encaminhar e prescrever. Precisa receber, colaborar no acolhimento amoroso, estabelecer confiança, distribuir qualidade independente da quantidade, desenvolver um ambiente promotor de estímulos positivos, renovar a comunicação interdisciplinar, participar das avaliações de desenvolvimento do bebê, reforçar a relação mãe/pai/bebê, mostrar os achados positivos nos retornos, informando os pais por meio de uma linguagem simples do primeiro ao último retorno quando da alta da terceira etapa.

Com muita clareza, registramos que a intervenção precoce que realizamos através do MC é fundamental para exercermos o cuidado adaptativo, permitindo aos pais expressarem e sobreporem seus conflitos, preocupações, medos, inseguranças e o próprio comportamento imaturo do recémnascido pré-termo e/ou de baixo peso.

A terceira etapa do MC nos permite um feedback fantástico das etapas anteriores e remete pais e bebês envoltos em maior segurança para o acompanhamento no ambulatório de pediatria. A visita domiciliar pode contribuir para o apoio à família, e sua inserção na terceira etapa do MC deve transcender à ausência da mãe e do bebê nos retornos agendados, fato não ocorrido neste estudo.

Finalmente, esperamos ter contribuído com um enfoque diferenciado sobre a literatura existente sobre a metodologia canguru, pois, abordando especificamente a vivência da terceira etapa, teremos um recorte de preposições teórico-práticas que desafiam o ideal da humanização.

A importância de novos estudos em seguimento ambulatorial contribuirá para melhor inserção da terceira etapa do MC nas instituições de saúde, pois é nesta que estaremos conduzindo bebês, mães e familiares para apurarem seus cuidados, habilidades e segurança no acompanhamento do desenvolvimento e crescimento, trazendo ao nosso conhecimento riscos de alterações precoces para controles especiais e encaminhamentos necessários na rede básica de referência.

\section{REFERÊNCIAS}

1. Ministério da Saúde(BR). Atenção humanizada ao recém-nascido de baixo peso. Método Canguru: manual técnico. Brasília(DF); 2011.

2.Moreira MEL, Lopes JMA, Carvalho M. 0 recém-nascido de alto risco: teoria e prática do cuidar. Rio de Janeiro: Fiocruz; 2004. (Col. Criança, Mulher eSaúde).

3.Guimarães GP, Marisa M. A formação do apego pais/recém-nascido pré-termo e/ou baixo peso no método mãe-canguru: uma contribuição da enfermagem. Texto Contexto \& Enferm. 2007 out/dez; 16(4).

4.Cabral IE, Rodrigues EC. 0 método mãe-canguru em uma maternidade do Rio de Janeiro 2000-2002: necessidades da criança e demanda de educação em saúde para os pais. Texto Contexto \& Enferm. 2006 out/ dez;15(4).

5.Brofenbrenner U. A ecologia do desenvolvimento humano e experimentos naturais planejados. Porto Alegre: Artes Médicas; 1996.

6.Charpak N. Kangaroo mother care in developing countries. 5th World Congress of Perinatal Medicine; 2001.

7.Nobre FDA, et al. Risco neonatal e desenvolvimento infantil avaliado através da "Escala de desenvolvimento do comportamento da criança: primeiro ano de vida -EDCC. Rev Paul Pediatr .2004; 22(35).

8.Oliveira MCB. Avaliação do crescimento de recém-nascidos de risco egressos de UTIs neonatais: um estudo prospectivo e multicêntrico [dissertação de mestrado]. Rio de Janeiro: Fiocruz; 2002.

9. Holanda ACOS, Silva MGC. Caracterização dos recém-nascidos de muito baixo peso egressos de uma UTI neonatal. Rev Paul Pediatr. 2004; 22(37). 
10.Toma TS. Método mãe-canguru: o papel dos serviços de saúde e das redes familiares no sucesso do programa. Cad Saude Publica. 2003 maio; 19(2).

11.Arruda DC, Marcon SS. A familia em expansão: experenciando intercorrências na gestação e no parto ao bebê prematuro com muito baixo peso. Texto\&Contexto Enferm. 2007 jan/mar; 16(4).

12. Tronchin DMR, Tsunechiro MA. A experiência de tornarem-se pais de prematuros um enfoque tecnológico. Rev Bras Enferm. 2005 jan/fev; 58(1).

13.Roy SC. Introduction to nursing an adaptation model. New Jersey: Prentice-Hall, Englewood Cliffs; 1984.

14.Trentini M, Paim L. Pesquisa convergente-assistencial: um desenho que une 0 saber fazer e o saber pensar na prática assistencial em saúdeenfermagem. $2^{\mathrm{a}}$ ed. Florianópolis: Insular; 2004.

15.Minayo MCS. Pesquisa social: teoria método e criatividade. $9^{a}$ ed. São Paulo: Vozes; 1994

16.Silva FS, Santos I. Expectativas de familiares de clientes em UTI sobre o atendimento em saúde: estudo sociopoético. Esc Anna Nery. 2010 abrjun; 14(2): 230-235.

17.Maas T. 0 processo de transição do ser adolescente hospitalizado com doença crônica sob a ótica da Enfermagem [dissertação de mestrado]. Curitiba:. Programa de Pós-Graduação em Enfermagem/ UFPR; 2006.

18.Silva ப, Silva LR, Christoffel MM. Tecnologia e humanização na unidade de terapia intensiva neonatal: reflexões no contexto do processo saúdedoença. Rev Esc Enferm USP. 2009 set; 43(3). 